\title{
3D Road Environment Modeling Applied to Visibility Mapping: an Experimental Comparison
}

\author{
Jean-Philippe Tarel ${ }^{*}$, Pierre Charbonnier ${ }^{\dagger}$, François Goulette ${ }^{\ddagger}$, and Jean-Emmanuel Deschaud ${ }^{\ddagger}$ \\ *Université Paris Est, LEPSiS, IFSTTAR \\ Paris, France \\ Email: jean-philippe.tarel@ifsttar.fr \\ †CETE de l'Est, ERA 27 IFSTTAR, \\ Strasbourg, France \\ Email: Pierre.Charbonnier@developpement-durable.gouv.fr \\ $\ddagger$ Centre de Robotique - CAOR, Mines-Paris Tech, \\ Paris, France \\ Email: \{francois.goulette,jean-emmanuel.deschaud\}@mines-paristech.fr
}

\begin{abstract}
Sight distance along the pathway plays a significant role in road safety and in particular, has a clear impact on the choice of speed limits. Mapping visibility distance is thus of importance for road engineers and authorities. While visibility distance criteria are routinely taken into account in road design, few systems exist for evaluating them on existing road networks. Most available systems comprise a target vehicle followed at a constant distance by an observer vehicle. This only allows to check if a given, fixed visibility distance is available: estimating the maximum visibility distance requires several passages, with increasing inter-vehicle intervals. We propose two alternative approaches for estimating the maximum available visibility distance, that exploit 3D models of the road and its close environment. These methods involve only one acquisition vehicle and use either active vision, more specifically 3D range sensing (LIDAR), or passive vision, namely, stereovision. The first approach is based on a Terrestrial LIDAR Mobile Mapping System. The triangulated 3D model of the road and its surroundings provided by the system is used to simulate targets at different distances, which allows for estimation of the maximum geometric visibility distance along the pathway in a quite flexible way. The second approach involves the processing of two views taken by digital cameras on-board an inspection vehicle. After road segmentation, the 3D road model is reconstructed which allows maximum roadway visibility distance estimation. Both approaches are described, evaluated and compared. Their pros and cons with respect to vehicle-following systems are also discussed.
\end{abstract}

\section{INTRODUCTION}

In this paper, we address the problem of mapping the visibility distance along an existing itinerary, using 3D models of the road and its environment.

It may be noticed that visibility distance is a complex notion and has many different definitions in the specialized literature. All of them, however, are functional. In other words, they depend on a scenario of usage, e.g. overtaking a vehicle or approaching key roadway features. Among the many definitions that can be found, we consider the stopon-obstacle scenario, which leads to the notion of stopping sight distance (SSD) [1]. More specifically, we define the required visibility distance as the distance needed by a driver to react to the presence of an obstacle on the roadway and to stop the vehicle. This distance clearly depends on factors such as the driver's reaction time and the pavement skid resistance. These can be set to conventional, worst-case values, e.g. 2 seconds for the reaction time and a value corresponding to a wet road surface for skid resistance. Naturally, the stopping distance also depends on the speed of the vehicle, whose value may be inferred by modulating the so-called $V_{85}$ speed, defined as the 85th percentile of the speed statistical distribution. To this end, we use the geometrical characteristics of the road, namely curvature and slope, according to the same laws as for road design [1], [2]. The required visibility distance has to be compared to the available visibility distance, which is the highest distance at which an object can be seen on the road as a function of the geometry of the road environment.

Sight distance clearly plays a crucial role in the driving task. Visibility is then of importance as well for road engineers and managers, whose responsibility is to design or improve their network to ensure the user's safety, as for authorities, that must enforce credible and efficient speed limits. While visibility criteria are routinely taken into account in road design [1], [2], they are seldom evaluated on existing road networks. One reason for that might be that available evaluation systems are rather restrictive. Most of them operate in situ. They require two vehicles: a target vehicle followed at a fixed distance by an observer vehicle. A human operator, on board the observer vehicle, indicates at every moment whether the target vehicle is visible or not, which is obviously subjective. Moreover, such a set-up only allows to check that a given, fixed, sight distance is available. Estimating the maximal available visibility distance requires several passages, with stepwise increasing vehicle intervals, which can quickly become awkward. 


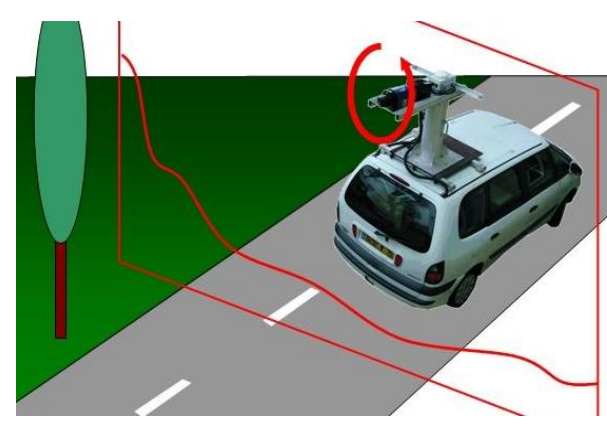

(a)

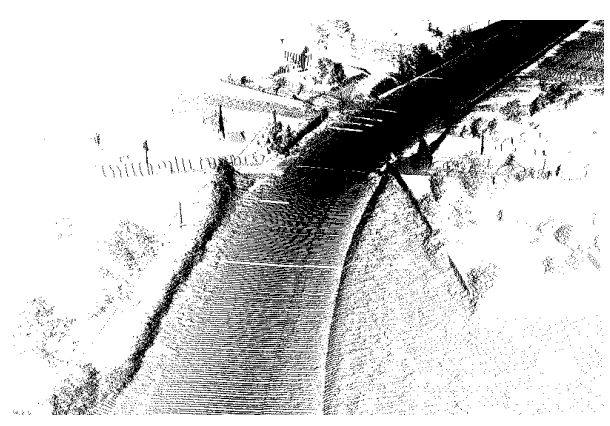

(b)

Figure 1. (a) The experimental vehicle, LARA3D; (b) an example of obtained 3D point cloud.

We believe that using 3D models offer an interesting alternative to vehicle-following systems, by providing more flexible, accurate and objective visibility assessment tools. In this paper, we investigate the use of two kinds of such numerical models for visibility estimation. To our knowledge, no such system was previously reported in the literature, excepted from a recent work, based on airborne LIDAR data [3]. In contrast, we consider terrestrial sensors, based on either active or passive vision technologies.

More specifically, we first investigate (Sec. II) the use of 3D data provided by a LIDAR terrestrial Mobile Mapping System. As the inspection vehicle moves, 3D points are sampled along the road and registered in an absolute reference system thanks to the use of INS and GPS sensors. The resulting point cloud is processed off-line to construct a triangulated 3D model of the road and its environment. Traditional visualization techniques such as ray-tracing or z-buffering are then used to implement the computation of available visibility.

Since road visibility evaluation by drivers is essentially a visual task, the second approach we propose (Sec. III) exploits images taken by cameras mounted on an inspection vehicle. Using two cameras allows modeling the 3D shape of the road, through stereovision. This model is exploited in conjunction with the results of an original roadway segmentation technique to evaluate the available visibility of the road.

Evaluating such systems on existing roads is not an obvious task due to the difficulty of establishing a reliable ground-truth. We have chosen to consider an operational vehicle-following system as a reference. We provide experimental comparisons on both a test-track and a trafficked road section (Sec. IV).

We close the paper (Sec. V) by a discussion about the pros and cons of the proposed methods and working perspectives.

\section{MobILE LIDAR-BASED METHOD}

In this approach, we build a 3D model of the road environment with a mobile mapping system based on LIDAR sensors. Then, we compute the visibility distance with a software ray-tracing algorithm directly on $3 \mathrm{~d}$ mesh. We will detail in this section all processes (system, modeling and distance computation) specific to the application of visibility distance estimation.

\section{A. Active sensor acquisition system}

Our mobile mapping system, called LARA3D, is a prototype with the following sensors: positioning sensors (GPS and IMU), range sensors (two SICK laser scanners) and photogrammetric sensors (Pike and Canon cameras). The location of the vehicle is calculated using a Kalman Filter with GPS/IMU inspired from [4]. The laser scanners are installed on the roof of the vehicle at about $2.7 \mathrm{~m}$ height to scan a plane perpendicular to the driving direction. Knowing the geographic location of the vehicle, we register the successive laser cross sections obtained over the vehicle's passage like Fig. 1. This provide coverage of roads and their surroundings (buildings, vegetation) in the form of a dense 3D point cloud as seen on Fig. 1. The number of points per kilometer depends on the vehicle's speed: at $50 \mathrm{~km} / \mathrm{h}$, we have around 2000000 points per kilometer.

\section{B. 3D Road environment modeling}

The next step is to build a realistic 3D model of the environment from this 3D point cloud. Modeling the environment from raw laser data is done by following a set of processes. Here is an overview of the processes applied to our laser data: automatic removal of artifacts in laser cross sections, decimation of neighborhood points, denoising, meshing, detection of flat areas and model simplification. We detail each of these processes in the following sections.

1) Automatic removal of artifacts: When scanning roads, the vehicle is driving in normal traffic. Others cars or trucks are scanned within the environment, causing artifacts. If these artifacts are not removed, they lead to false objects in the environment and thus to mistakes in the calculation of visibility distances. We have developed an automatic method to remove artifacts on the road. To this end, we process directly each laser cross section containing a cross section of the road. Indeed, knowing parameters like the height of the 

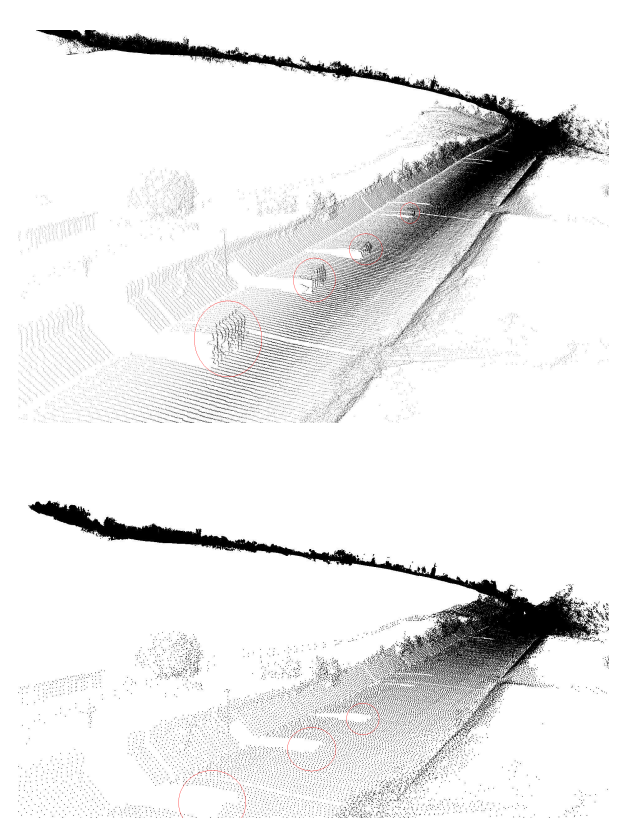

Figure 2. The automatic removal of artefacts in 3D point clouds.

laser and the distances of the vehicle to the roadside on the right and on the left of the vehicle, the points out of the road are removed, as illustrated in Fig. 2. One limitation of this method is to be dependent on the geometrical configuration of the laser: it works only if there is road data points in the laser cross section.

2) Decimation of neighborhood points: Raw point clouds previously obtained are not uniformly distributed over the surfaces to be reconstructed. This non-uniform distribution of points does not allow proper calculation of the normals, when correct normals are required to obtain a triangulation without elongated triangle. To avoid this, we perform a decimation of points per neighborhood: if two points are closer than some distance $d$, we remove one of the two points. This distance $d$ will depend on the resolution of data in the laser cross section (which depends on the angular resolution of laser and on the distance of the point to the laser) and on the resolution of data along the trajectory (which depends on vehicle speed and on the frequency of the scanner). Best sampling is achieve when it is uniform in these two directions.

3) Denoising and Meshing: Once the point cloud is decimated, we can build the 3D mesh model which is subject to the presence of noise in the point cloud. It is therefore necessary to go through a denoising step, prior to the triangulation. For data denoising, we used the image processing method called "Non Local Denoising" [5]. This denoising method works directly on a point cloud and does not require meshing. The Fig. 3 shows the 3D models with and without the denoising step, with meshing in order to facilitate the visualization. After denoising, the mesh is build
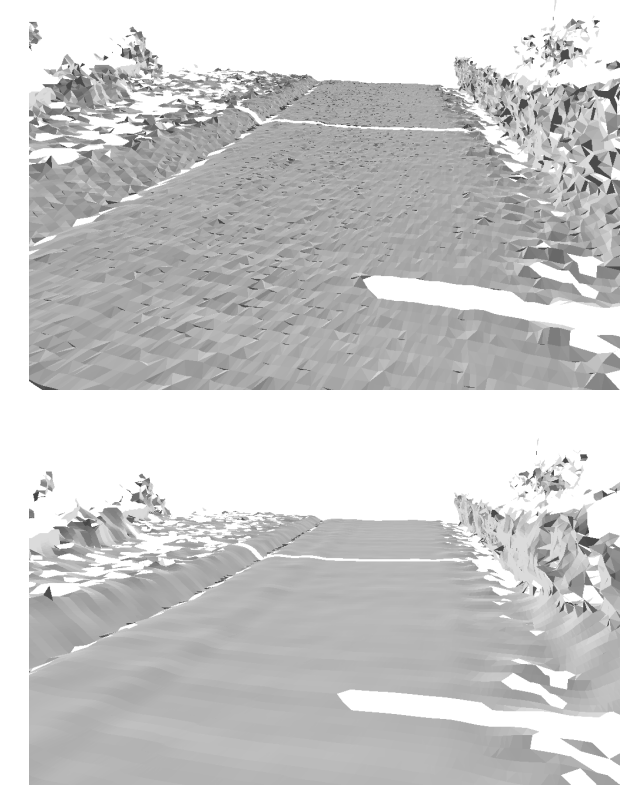

Figure 3. The meshing without and with denoising step.

using a triangulation method which is a variant of the Ball Pivoting Algorithm (BPA) [6].

4) Detecting flat areas and simplification of the model: After meshing, the obtained 3D models contain around two millions of triangles per kilometer. As the ray-tracing algorithm for visibility distance estimation is very slow and its speed depends on the number of triangles, we need to simplify the model. We used the fast algorithm for detecting flat areas in large point clouds described in [7] and then apply a classical simplification method based on [8] in nonflat area. These two kind of simplifications leads to arround 200000 triangles per kilometer.

5) The modeling pipeline: We described the different steps of the processing from the point cloud to the simplified and sufficiently realistic 3D model of the road environment. All processes (decimation, denoising, meshing and simplification) are done off-line but in a time lower that the acquisition time. As consequence, it would be possible to directly produce the final triangulated model, without raw data recording. Fig. 4 shows two examples of obtained 3D models.

\section{Estimating the visibility distance from a $3 D$ model}

The definition of the available visibility distance we use in this approach is purely geometric and does not involve any photometric or meteorological consideration. It involves a target and an observer. The target may either be placed at a fixed distance from the observation point, to assess the availability of a specific distance, or it may be moved away from the observation point until it becomes invisible, to estimate the maximum visibility distance. Conventional values can be found in the road design literature, see [2], 


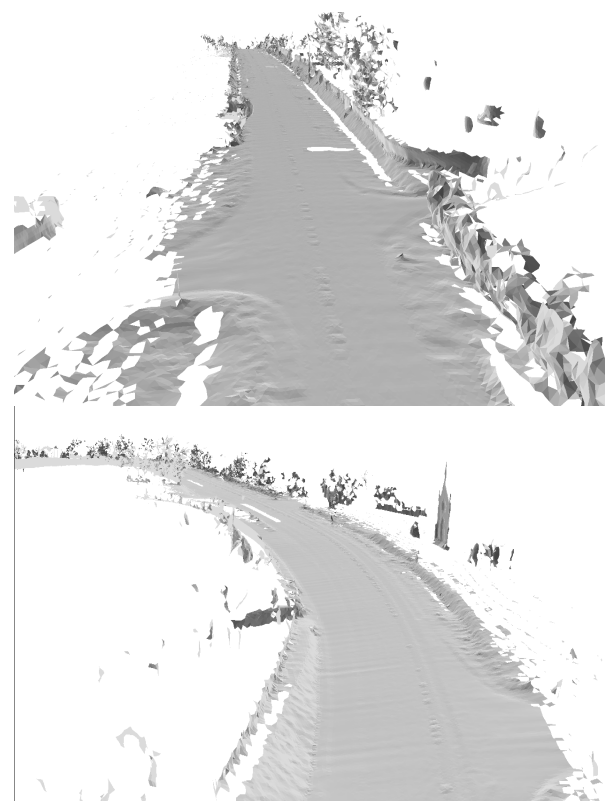

Figure 4. Two examples of obtained 3D models of the road environment used for visibility distance estimation.

for both the location of the observer and the geometry and position of the target. Both the viewpoint and the target are centered on the road lane axis. Typically, the viewpoint is located $1 \mathrm{~m}$ high, which roughly corresponds to a mean driver's eye position. The conventional target is a pair of points that model a vehicle's tail lights. For visibility distance computation, we require a line-of-sight connection between the tail lights and the driver's eyes. Ray-tracing algorithms are well-suited for this task. We also found it realistic to consider a parallelepiped $(1.5 \times 4 \times 1.3 \mathrm{~m})$ to model a vehicle. We found that a good rule of thumb was to consider the target as visible if $5 \%$ of its surface is visible. The threshold was set experimentally, in such a way that the results of the test would be comparable to the ones obtained when using the conventional target.

We developed a specific software application (see Fig. 5), that allows to walk through the 3D model (which can be visualized as a point cloud or a surface mesh). The trajectory of the inspection vehicle on the road may also be visualized in the 3D model. Volumetric or point-wise targets may be placed at different distances from the viewpoint (see Fig. 6). To make the interpretation easier, images of the road scene may be visualized along the 3D model. All views are synchronized and the interface is completely reconfigurable.

The software implements both required and available visibility distance computation. For the point-wise, conventional target, we use a software ray-tracing algorithm. When the volumetric target is used, we exploit the Graphical Processing Unit's capabilities. More specifically, the target is first drawn in the graphical memory, then the scene is rendered using Z-buffering and finally, an occlusion-query request

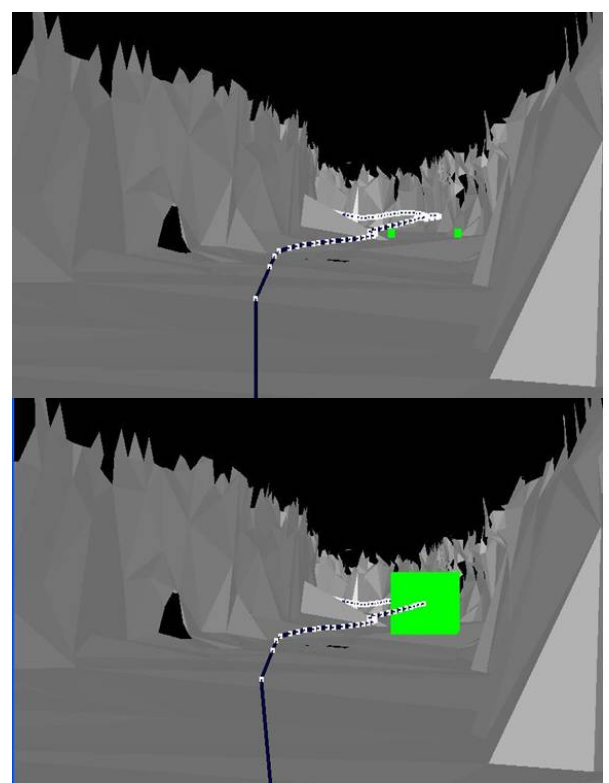

Figure 6. Example of 3D model (gray triangles) with targets (in green). Top: pairs of points representing tail lights; Bottom: volumetric target.

(which is standard in up-to-date OpenGL implementations) provides the percentage of visible target surface. The target is zoomed on and centered in the scene, to mimic the focusof-attention mechanism of a human observer.

The visibility distance can be computed at every point of the trajectory (i.e. every 1 meter) or with a fixed step (typically, 5 or 10 meters) to speed up computations. Two different ways of computing the visibility are implemented. In the first case, a fixed distance is maintained between the observation point and the target (as for vehicle-following systems). The output is a binary function which indicates at every position whether the prescribed distance is available or not. In the second case, for every position of the observation point, the target is moved away as long as it is visible and the maximum available visibility distance is recorded.

\section{MOBILE STEREOVISION METHOD}

The available visibility distance can be defined as the maximum distance of points belonging to the image of the road. This distance can be estimated with stereovision looking at the road ahead the vehicle. This distance is similar to the one perceived by the driver, when the distance between the driver's eye and the cameras is small.

\section{A. Passive sensor acquisition system}

A experimental inspection vehicle was developed for stereo pair of images acquisition. The images are acquired every 5 meters, independently of the vehicle speed. Preliminary to every acquisition, the stereo head is calibrated intrinsically and extrinsically with respect to the road using a grid painted on the road. The acquired images are rectified 


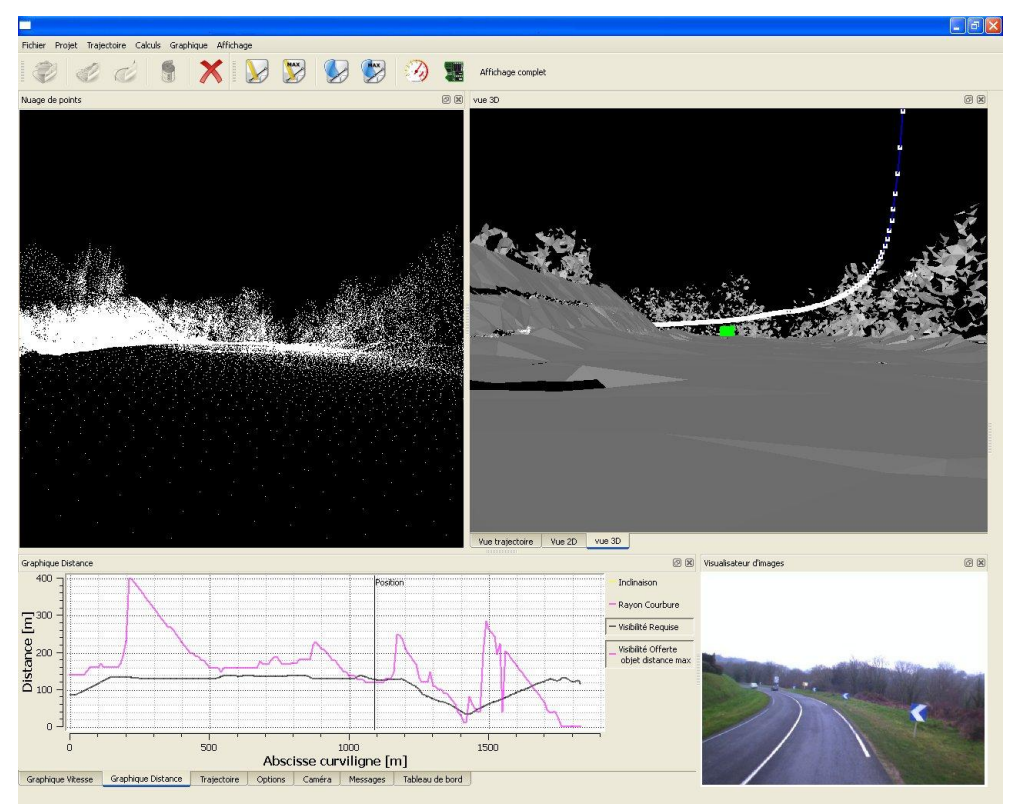

Figure 5. Interface of the sight distance computation software. The top-left panel displays the original point cloud. The top-right panel shows the triangulated model (in grey), the trajectory of the LIDAR system (in blue) and a parallelepiped target placed $100 \mathrm{~m}$ ahead of the observation point (in green). At the bottom right, an image of the scene is displayed. Finally, the bottom-left panel displays the required visibility (black) and the estimated available visibility (magenta) vs. the curvilinear abscissa. The vertical line shows the current position. The curves show that the available visibility is not sufficient in this situation.

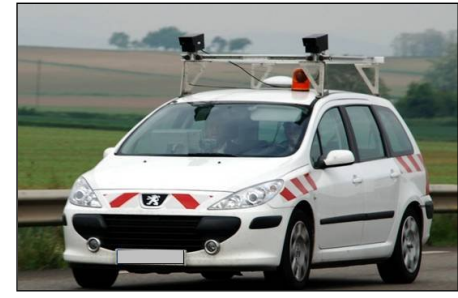

(a)

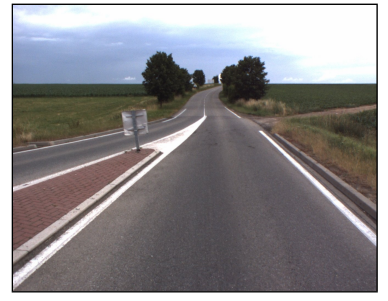

(b)

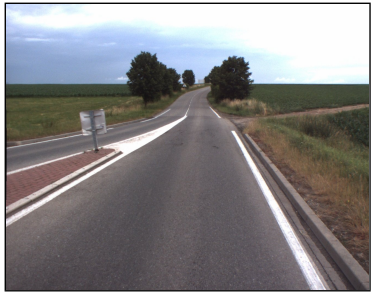

(c)

Figure 7. (a) Experimental inspection vehicle with two digital cameras for stereovision; (b),(c): stereo pair of images acquired by the vehicle.

and corrected of the geometrical distortions of the lenses. Lens vignetting is also corrected.

\section{B. Local road modeling}

On each of the rectified stereo pair, a two-step processing is performed to obtain the local road model: segmentation of the roadway on left and right images using color information, 3D reconstruction of the edges in the road regions of the left and right images allowing to estimate a 3D parametric model of the road surface.

The first step of the processing consists in classifying each pixel, in left and right images, as road or non-road. Following the segmentation approach proposed in [9], this segmentation consists on an iterative learning of the colorimetric characteristics of both the road and the non-road pixels, along the image sequence. To this end, pixels in the bottom center of each image are assumed to belong to the road class, while pixels in the top left and right regions are assumed non-road elements. These regions may be visualized in the left image of Fig. 8 . The algorithm being run in batch, the road and non-road color characteristics are collected in following images. The advantage is that it allows sampling road colors at different distances ahead of the current image. This strategy leads to improved segmentation results in the presence of lighting perturbations, shadows, variations of pavement color. Once the road and non-road color models are built, the image is segmented in two classes by a region growing algorithm, starting from the road (i.e. bottom center) part of the image. Fig. 8, center image, shows an example of segmentation result. To keep a correct road color model, occlusions of the road region can be filtered out by removing colors which are not usually found on the pavement.

The second step consists in extracting the edges in the left and right road regions segmented in the first step 


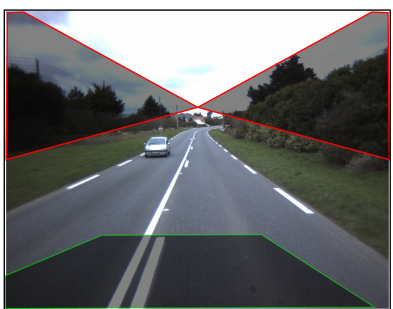

(a)

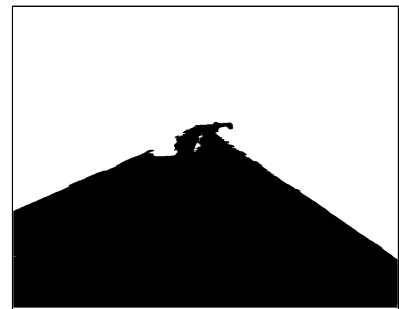

(b)

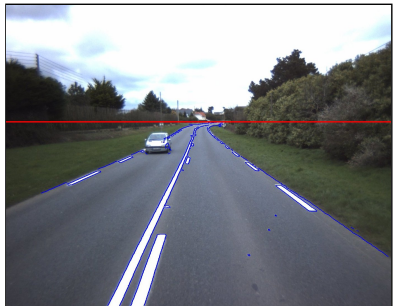

(c)

Figure 8. The original image (a) is processed to learn road colors from pixels in the green region, and non-road colours from pixels in the red region. (b) The resulting classification of pixels as road (black) and non-road (white) pixels. (c) The result of stereo matching: contours extracted from the right view of the stereo pair are back-projected and superimposed (in blue) on the left view. The maximum ahead visibility is shown as a red line.

and to reconstruct the surface modeling the road, thanks to correspondences between the left and right edges. We use a 3D model based reconstruction algorithm, originally proposed in [10]. Assuming a polynomial parametric model of the road longitudinal profile, this algorithm is based on an iterative scheme that alternates between matching of pairs of edge pixels and the estimation of road surface parameters. The higher the number of edges (even those due to dust on the road may be useful), the better the registration. Of course, occulted parts of the road can not be correctly reconstructed.

\section{Road visibility distance estimation}

Once the 3D surface of the road has been locally estimated, the maximum distance at which edges on the road surface can be found is estimated. An example of result is shown in Fig. 8 right image, where the red line corresponds to the visibility height in each image. The standard deviation of the estimator is also computed, in order to qualify this estimated visibility distance. A large stereo basis of 1 meter is used to have enough accuracy in the reconstruction at large distances.

\section{EXPERIMENTS AND COMPARISON WITH VISUAL REFERENCE}

Evaluating sight distance estimation methods is a rather difficult task because it is quite impossible to design a proper ground truth on an existing itinerary. We therefore chose to use the visual estimation provided by an operational vehiclefollowing system as a reference for our experiments.

\section{A. Vehicle-following reference}

The system is illustrated on Fig. 9. It consists of two vehicles, equipped with odometers and connected by radio in order to maintain a fixed distance between each other. An operator, in the observation vehicle, visually checks whether the leader, target vehicle is visible or not. These (binary) observations are recorded using an ad hoc software. In order to perform our experimental comparisons, we needed to estimate the maximum sight distance. Hence, the vehiclefollowing system had to be run with several intervals: 50,

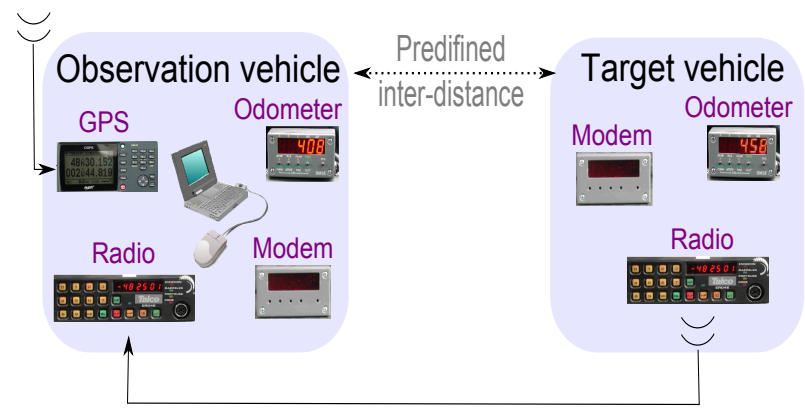

Figure 9. Operational vehicle-following sight distance evaluation system.

$65,85,105,130,160,200,250$ and 280 meters. The results of these individual experiments were then merged using the following rule to eliminate partial maskings: if the target is visible from 0 to some distance, say $d_{1}$, then masked until $d_{2}$ and visible again until $d_{3}$, then the maximal visibility is $d_{1}$, not $d_{3}$.

\section{B. Experimental Comparison}

The available visibility distances estimated using the three discussed techniques: vehicle following, stereovision, active $3 \mathrm{D}$ modeling and the required visibility are compared on a trafficked secondary road section of $2 \mathrm{~km}$ long and on a testtrack. The results are shown on Fig. 10 and 11, respectively. In both cases, the results of the vehicle-following sight distance evaluation system are shown as a green discontinuous curve. The required stopping sight distance, evaluated using the road characteristics is shown as a red, continuous curve. The estimated available visibility obtained using stereovision road modeling is displayed as a blue curve with pluses, and the black curve with stars shows the results of the approach based on active 3D modeling.

The results show that the stereovision approach underestimates the visibility distance over most of the length of the sections. A careful examination of the results has shown that, on the trafficked road, the algorithm is very sensitive to the presence of vehicles in the road scene. Moreover, the long range road/non-road classification becomes more difficult when colors are flatter, which occurs under certain 


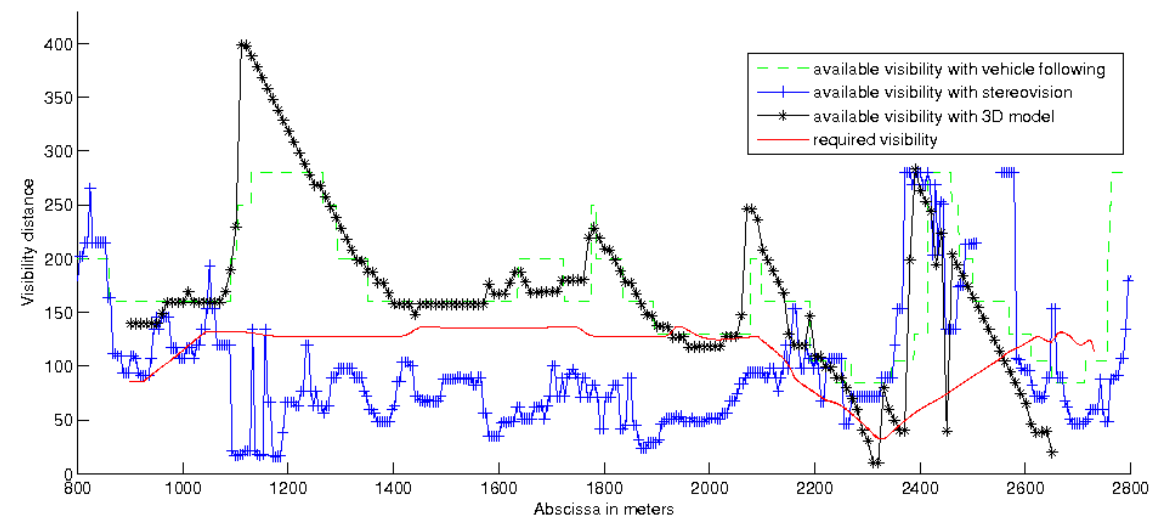

Figure 10. Comparison on the same $2 \mathrm{~km}$ road section, of the estimates of the available visibility distance using 3 techniques: vehicle following, stereovision, active $3 \mathrm{D}$ modeling. The required visibility computed from the road geometry is also displayed. When the available visibility is lower that the required one (e.g. at abscissa $2000 \mathrm{~m}$ ), a lack of visibility is detected.

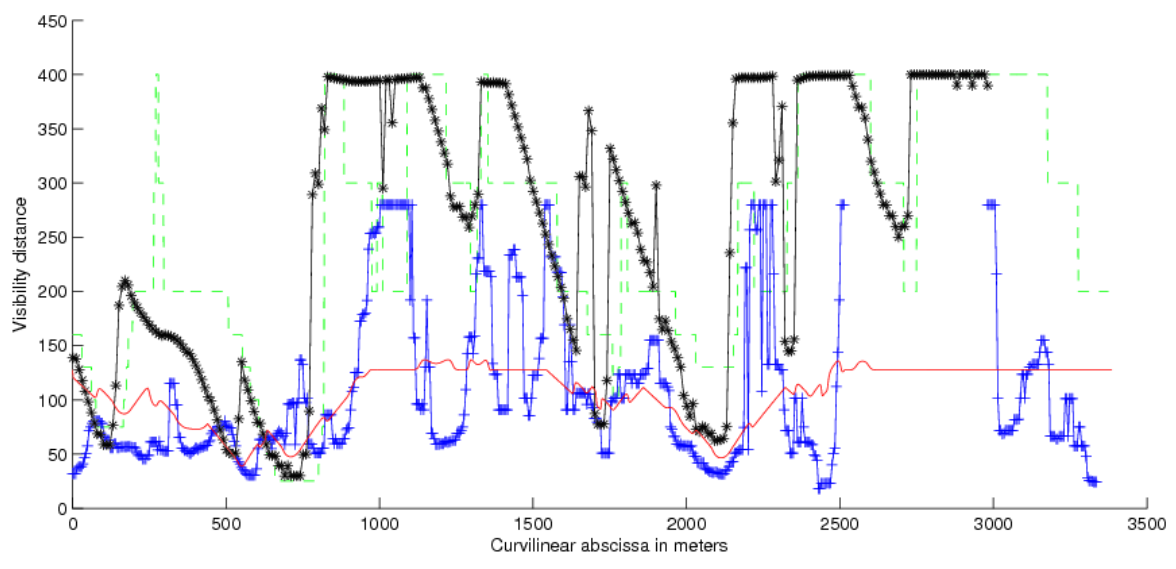

Figure 11. Comparison on the same $3 \mathrm{~km}$ road section, of the estimates of the available visibility distance using 3 techniques: vehicle following, stereovision, active 3D modeling. The required visibility computed from the road geometry is also displayed.

weather conditions or during the winter. We also noticed, on the test track, that in curves with a high curvature, the stereovision method is limited by the aperture angle of the optics which was limited with our system to achieve good accuracy at long distances.

In contrast, the results of the 3D-based method are globally in good accordance with those of the reference method. Certain differences are observable, anyway. This is illustrated in Fig. 12, where both the 3D model and the image of the scene, considered at the abscissa $1670 \mathrm{~m}$ on the test-track, are displayed. It can be seen that a target placed $300 \mathrm{~m}$ ahead the observation point is visible underneath the canopy of the trees, while the human observer recording a masking at this particular point.

Finally, let us note that in certain cases, the available sight distance is less than the required one. This occurs, for example, near the abscissa $2000 \mathrm{~m}$ on the trafficked secondary road section, see Fig. 10 (or abscissa $1100 \mathrm{~m}$ on Fig. 5). This means that there is a problem of sight distance at this point of the itinerary, due to the presence of an embankment on the left, which was confirmed by accidentology studies.

\section{CONClusion}

In this paper, we have proposed two methods that exploit 3D models of the road and its close surroundings, obtained either from active or by passive vision systems, to estimate the available visibility distance along existing roads. While limited, our experiments shows encouraging results. In particular, as soon as an accurate 3D model of the road and its surrounding is available, well-known computer graphics techniques such as ray-tracing or z-buffering seem sufficient to estimate the available sight distance. However, obtaining such models requires a careful processing of the point cloud. In particular, the triangulated model must be simplified enough to allow processing long road sections, but without losing useful information (especially when modeling vegetation). Note that this processing allows getting rid of 


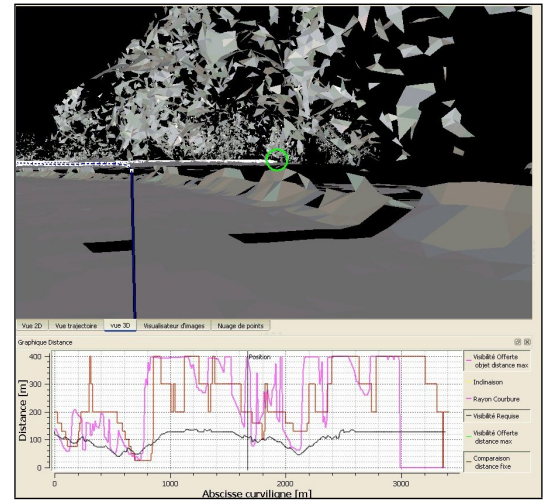

(a)

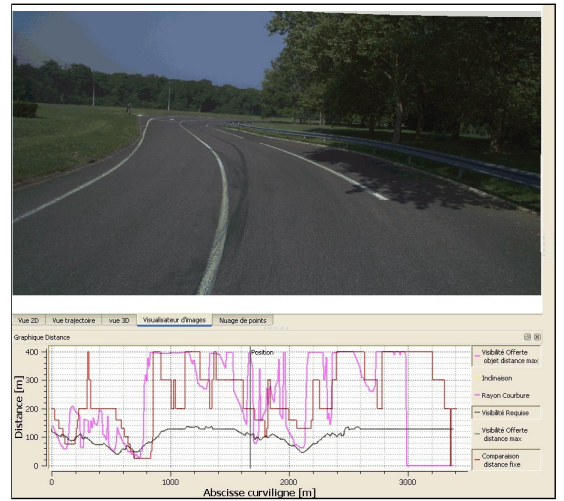

(b)

Figure 12. View of the 3D model (a) and the image of the scene (b) at abscissa $1670 \mathrm{~m}$ on the test track. In the $3 \mathrm{D}$ model, the trajectory is show as a blue line with white dots. A volumetric target placed at $300 \mathrm{~m}$ ahead the observation point is visible in the green circle. At the bottom of each image, sight visibility distances are show: in black, required visibility; in purple, available visibility estimated from the active $3 \mathrm{D}$ model; in light brown, available visibility using the vehicle-following system.

false detections due to the traffic and eliminating scanning artifacts, in general.

On the other hand, the method based on stereovision and image processing tends to underestimate the visibility distance due to the difficulties inherent to the task of segmenting the road far ahead the camera, and to the limited field of view of the stereo rig in sinuous sections. The method is also sensitive to occlusions due to traffic. Stereovision, however, could be used as a cheap 3D sensor in the near field.

Future working directions involve further validation experiments as well as improvements of the $3 \mathrm{D}$ method. In particular, image segmentation should be used to provide a better localization of the mobile and interpretation of the scene: for the moment, the trajectory of road is assimilated to the one of the acquisition vehicle, and only 2-tracks roads are considered. Extensions to highway environments might be considered since, for example, the visibility of the approaches of work areas is an important road safety issue. Finally, to be routinely used, such methods require that the scalability problem be solved, which remains a challenging issue.

\section{ACKNOWLEDGEMENTS}

The authors are thankful to the French PREDIT SARIVIZIR and ANR-DIVAS projects for funding. The VISULINE data was provided by the LRPC St Brieuc (ERA 33 LCPC).

\section{REFERENCES}

[1] M. Wooldridge, K. Fitzpatrick, D. Harwood, I. Potts, L. Elefteriadou, and D. Torbic, Geometric design consistency on high-speed rural two-lane roadways. Washington, DC : Transportation Research Board, National Research Council, 2003, vol. NCHRP Report 502.
[2] SETRA, ARP : Aménagement de Routes Principales (sauf les autoroutes et routes express à deux chaussées). SETRA, Aug. 2000, (in French).

[3] P. Bartie and M. Kumler, "Route ahead visibility mapping: A method to model how far ahead a motorist may view a designated route," Journal of maps, pp. 84-95, 2010, online, http://www.journalofmaps.com.

[4] F. Goulette, F. Nashashibi, I. Abuhadrous, S. Ammoun, and C. Laurgeau, "An integrated on-board laser range sensing system for on-the-way city and road modelling," in ISPRS RFPT, 2006.

[5] A. Buades, B. Coll, and J. Morel, "Nonlocal image and movie denoising," International Journal of Computer Vision, vol. 76, no. 2, pp. 123-139, Feb. 2008.

[6] F. Bernardini, J. Mittleman, H. Rushmeier, C. Silva, and G. Taubin, "The ball-pivoting algorithm for surface reconstruction," IEEE Transactions on Visualization and Computer Graphics, vol. 5, pp. 349-359, 1999.

[7] J.-E. Deschaud and F. Goulette, "A fast and accurate plane detection algorithm for large noisy point clouds using filtered normals and voxel growing," in Proceedings of $3 D$ Processing, Visualization and Transmission Conference (3DPVT2010), 2010.

[8] M. Garland and P. S. Heckbert, "Surface simplification using quadric error metrics," in Proceedings of the 24th annual conference on Computer graphics and interactive techniques. ACM Press/Addison-Wesley Publishing Co., 1997, p. 216.

[9] J.-P. Tarel and E. Bigorgne, "Long-range road detection for off-line scene analysis," in Proceedings of IEEE Intelligent Vehicle Symposium (IV'2009), Xian, China, 2009, pp. 15-20.

[10] J.-P. Tarel, S.-S. Ieng, and P. Charbonnier, "Accurate and robust image alignment for road profil reconstruction," in Proceedings of IEEE International Conference on Image Processing (ICIP'07), vol. V, San Antonio, Texas, USA, 2007, pp. 365-368. 